Gefässchirurgie $2020 \cdot 25: 446-449$ https://doi.org/10.1007/s00772-020-00665-w Online publiziert: 16 . Juli 2020

(c) Der/die Autor(en) 2020
Johannes Klopf · A. Scheuba · C. Brostjan · C. Neumayer · W. Eilenberg

Universitätsklinik für Chirurgie, Klinische Abteilung für Gefäßchirurgie und chirurgische Forschungslaboratorien, Medizinische Universität Wien, Allgemeines Krankenhaus der Stadt Wien, Wien, Österreich

\title{
Bisherige Strategien und zukünftige Perspektive zur Reduzierung der Wachstums- raten bei abdominellen Aortenaneurysmen
}

\section{Eine selektive Literaturrecherche sowie Erörterung des aktuellen Vienna MetAAA Trials}

\section{Bisherige Forschungs- ergebnisse}

In der Medizingeschichte sind arterielle Aortenaneurysmen seit über 3500 Jahren bekannt. Seit dem Papyrus Ebers, der überlieferten erstmaligen Schrift über das menschliche Herz, Aorta und Aneurysmen, stellt sich die Frage nach einer optimalen Therapie des abdominellen Aortenaneurysmas (AAA). Wurden im alten Ägypten noch magische Heilmittel verabreicht, stehen heutzutage bereits moderne endovaskuläre Techniken zur chirurgischen Therapie zur Verfügung [1]. Oft ist die entscheidende Frage bei Patienten mit AAA allerdings, wie lange man den Zeitpunkt, bis eine operative Versorgung unumgänglich ist, hinauszögern kann. Der folgende Artikel gibt einen Überblick zu bisherigen therapeutischen Strategien und Empfehlungen zur medikamentösen Therapie. Der Fokus liegt hierbei auf klinischen Studien zu Arzneimittelgruppen, welche auf ihre Wirksamkeit hin untersucht wurden, die Wachstumsrate von abdominellen Aneurysmen zu verringern. Als Beispiel einer aktuell laufenden Studie wird das Vienna MetAAA Trial vorgestellt.
Für das Ziel, die Progression oder die Ruptur eines AAA zu vermeiden, erscheint zunächst die konsequente Therapie der behandelbaren Risikofaktoren naheliegend. Der negative Einfluss von gesundheitsschädlichem Rauchverhalten und die zudem klar positive Assoziation der Dosis-Wirkungs-Beziehung zur AAA-Wachstumsrate steht wissenschaftlich außer Frage. Die Aufgabe des Rauchverhaltens kann bei Patienten die AAAWachstumsrate um bis zu $20 \%$ reduzieren [2-4]. Eine randomisiert kontrollierte Studie sowie eine rezent durchgeführte Metaanalyse in Bezug auf die Sicherheit und Wirksamkeit von Bewegungstraining bei AAA-Patienten zeigten keinen Einfluss auf die AAA-Wachstumsrate und weder der Trainingsstatus noch das Fitnessniveau beeinflussten die AAAProgression signifikant. Angeleitetes Bewegungstraining zeigte sich zumindest bei Patienten mit AAA im Allgemeinen als sicher $[5,6]$.

Im Jahr 2015 lieferte eine systematische Übersichtsstudie eine evidenzbasierte Zusammenfassung der Daten bezüglich pharmakologischer Thera- piemöglichkeiten zur Stabilisierung oder zukünftigen Unterbindung des AAA-Wachstums und schlussfolgerte, dass zurzeit nur unzureichende Evidenz vorliegt und keine bisherig verfügbare medikamentöse Intervention das AAAWachstum verringern kann [7]. Trotz umfassender, weltweiter Forschungsanstrengung konnte bis heute keine Arzneimittelgruppe oder einzelnes Medikament mit der Fähigkeit zur Wachstumsratenreduktion beim abdominellen Aortenaneurysma identifiziert werden. Diese bisherigen Strategien haben initial

\begin{tabular}{|ll}
\hline \multicolumn{2}{|l}{ Abkürzungen } \\
\hline$A A A$ & Abdominelles Aortenaneurysma \\
\hline$A C E$ & Angiotensin Converting Enzyme \\
\hline ADAM & $\begin{array}{l}\text { Aneurysm Detection and Manage- } \\
\text { ment }\end{array}$ \\
\hline ESVS & $\begin{array}{l}\text { European Society for Vascular } \\
\text { Surgery }\end{array}$ \\
\hline $\begin{array}{ll}\text { HMG-CoA- } \\
\text { Reduktase }\end{array}$ & Coenzym-A-Reduktase \\
\hline LDL-C & $\begin{array}{l}\text { Low-density-Lipoprotein-Choles- } \\
\text { terin }\end{array}$ \\
\hline MMP-9 & Matrixmetalloproteinase 9 \\
\hline UKSAT & UK Small Aneurysm Trial \\
\hline
\end{tabular}


vielversprechende therapeutische Daten und Berichte gemein, die sich nachfolgend in größer angelegten Studien nicht bestätigen konnten. Im Spektrum der bereits untersuchten Arzneimittel liegen verschiedene Klassen an antihypertensiven Medikamenten wie BetaAdrenozeptor-Antagonisten, ACE-Inhibitoren, Kalziumkanalantagonisten und Diuretika. So konnte bei AdrenozeptorAntagonisten wie zum Beispiel Propanolol weder in Fall-Kontroll- oder Kohortenstudien noch in randomisierten, kontrollierten Studien ein signifikanter Effekt auf das AAA-Wachstum festgestellt werden [8-13]. Ebenfalls konnte für ACE-Inhibitoren kein positiver Effekt auf die AAA-Wachstumsrate nachgewiesen werden $[10,14,15]$. Eine prospektive Kohortenstudie mit 1701 Patienten zeigte sogar eine Assoziation zwischen der Einnahme von ACE-Inhibitoren und schnellerem AAA-Wachstum [16]. Die Hypothese, dass AAA-Wachstum in der Regel mit Infektionen zusammenhängen könnte und eine entsprechende antimikrobielle Therapie hier vorteilhaft wäre, wurde in Studien mittels Makroliden wie Roxithromycin und Azithromycin widerlegt [17, 18]. Auch Tetrazykline, insbesondere Doxycyclin, von dem berichtet wurde, dass es eine signifikante Reduktion von pathophysiologisch wichtiger MMP-9-Aktivität bedingt, konnten keine AAA-Wachstumsratenreduktion zeigen $[7,19,20]$. Die Wirkung von HMG-CoA-Reduktase-Inhibitoren (Statinen) auf das AAAWachstum ist aufgrund unterschiedlicher Studienergebnisse nicht eindeutig und wird durchaus kontrovers diskutiert $[21,22]$. Drei Kohortenstudien und eine klinische Studie bezüglich Azithromycin demonstrierten, dass die Verwendung von Statinen mit der Reduktion von AAA-Wachstumsraten assoziiert ist. Die durchschnittliche Verringerung des AAA-Wachstums in Patienten mit Einnahme von Statinen betrug zwischen 0,9 und $1,2 \mathrm{~mm}$ pro Jahr [23-26]. Allerdings konnten vier andere Studien mit höherer Fallzahl (zwei prospektive und zwei retrospektive Kohortenstudien) diesen Effekt nicht zeigen [16, 27-29]. Eine Metaanalyse folgerte, dass die Wirkung der Statintherapie auf die
Wachstumsrate von der Größe des Aneurysmas abhängt und vorteilhafter ist, wenn der AAA-Durchmesser $\geq 36 \mathrm{~mm}$ beträgt [30]. Eine sehr rezente Metaanalyse im Jahr 2020 untersuchte 14 Studien mit insgesamt 38.749 AAA-Patienten, davon unterliefen 15.993 Patienten eine Therapie mit HMG-CoA-ReduktaseInhibitoren, 22.756 Patienten erhielten ein Placebo. Die gepoolten Ergebnisse zeigten, dass die Einnahme von Statinen mit einer signifikant niedrigeren AAA-Wachstumsrate zusammenhängt. Der mittlere Unterschied zwischen den beiden Gruppen betrug 1,5 mm pro Jahr [31]. Aufgrund der Heterogenität der genannten und vielen anderen Studien findet sich zurzeit in den aktuellen Clinical Practice Guidelines der European Society for Vascular Surgery (ESVS) als auch in den Practice Guidelines der Society for Vascular Surgery keine Empfehlung zur medikamentösen Therapie mit HMG-CoA-Reduktase-Inhibitoren zum alleinigen Grund der AAA-Wachstumsratenreduktion [4, 32]. Allerdings erhalten AAA-Patienten Statine weitgehend, da diese indiziert sind, um das allgemeine kardiovaskuläre Risiko in AAA-Patienten zu senken. Dieser Umstand erschwert auch zukünftige Studien bezüglich der Wirkung von Statinen auf die AAA-Wachstumsrate. Die European Guidelines on cardiovascular disease prevention in clinical practice und die Guidelines on the Diagnosis and Treatment of Peripheral Arterial Diseases empfehlen bei allen Patienten mit peripher arterieller Gefäßerkrankung das Low-density-Lipoprotein-Cholesterin (LDL-C) auf $<1,8 \mathrm{mmol} / \mathrm{l}$ (<70 mg/dl) zu reduzieren oder um $50 \% \mathrm{zu}$ reduzieren, wenn der anfängliche LDL-C-Spiegel zwischen 1,8 und $3,5 \mathrm{mmol} / \mathrm{l}$ liegt (70 und $135 \mathrm{mg} / \mathrm{dl}$ ) $[33,34]$. Einige Studien untersuchten zudem das Potenzial von Thrombozytenaggregationshemmern, vor allem mittels Acetylsalicylsäure, als Therapie zur Stabilisierung des AAAWachstums. Eine dänische Studie mit 148 Patienten, welche ein AAA zwischen 30 und $48 \mathrm{~mm}$ aufwiesen, zeigte nach einer medianen Beobachtungszeit von 6,6 Jahren, dass in Patienten, deren AAA anfänglich $<40 \mathrm{~mm}$ Durchmesser hatte, eine ähnliche AAA-Wachstumsrate vorlag, unabhängig davon, ob Acetylsalicylsäure eingenommen wurde oder nicht. Allerdings zeigten abdominelle Aortenaneurysmen, die anfänglich bereits größeren Durchmesser als $>40 \mathrm{~mm}$ hatten, eine signifikante Reduzierung ihrer Wachstumsrate in Patienten, die Acetylsalicylsäure eingenommen hatten $(2,92 \mathrm{~mm} / \mathrm{Jahr}$ versus $5,18 \mathrm{~mm} / \mathrm{Jahr})$ [35]. Im Gegensatz dazu wurde im UK Small Aneurysm Trial (UKSAT), in der Aneurysm-Detection-and-Management(ADAM)-Studie und in einer weiteren Kohortenstudie kein signifikanter Effekt einer thrombozytenaggregationshemmenden Therapie auf die AAA-Progression festgestellt $[8,16,28]$. Bezüglich der Thrombozytenaggregationshemmer gelten zurzeit ähnliche Richtlinien wie für die antihypertensiven und lipidsenkenden Therapieansätze zur allgemeinen Reduktion des kardiovaskulären Risikos bei AAA-Patienten [4, 32]. Eine große Studie aus dem Jahr 2016 untersuchte verschiedene pharmakologische Therapien an 12.485 AAA-Patienten und konnte zeigen, dass die 5-Jahres-Überlebensraten signifikant erhöht waren für jene AAA-Patienten, die antihypertensive Medikation ( $62 \%$ versus $39 \%$ ), Statine ( $68 \%$ versus $42 \%$ ) oder thrombozytenaggregationshemmende Medikamente (64\% vs. $40 \%$ ) einnahmen, verglichen mit AAA-Patienten, die diese Arzneimittel nicht einnahmen [36].

Zusammenfassend besteht zurzeit keine ausreichende Evidenz für eine gültige Empfehlung laut internationalen Guidelines für Arzneimittelgruppen oder einzelne Medikamente, um die AAA-Progression zu verlangsamen oder gänzlich zu verhindern. Allerdings sollte ein gesunder Lebensstil einschließlich gesunder Ernährung und angeleitetem körperlichen Training sowie die medikamentöse Blutdruckkontrolle, lipidsenkende Therapie und Thrombozytenaggregationshemmung bei allen Patienten mit AAA in Betracht gezogen werden, falls keine Kontraindikationen vorliegen $[4,32]$. 


\section{Ausblick auf zukünftige Therapiemöglichkeiten}

Die Mehrzahl der bisher getesteten Medikamente zur Reduktion des Aneurysmawachstums ist auf Komorbiditäten ausgerichtet, denen man zum Teil überlappende Mechanismen der Pathogenese zuschreibt. So sind beispielsweise Hypertonie und Hyperlipidämie als Auslöser chronischer Entzündungsherde in der Atherosklerose bekannt, sind aber auch mit dem AAA assoziiert. Ein wirksames Therapeutikum zur Eindämmung der AAA-Progression sollte demnach der lokalen Zerstörung der Gefäßwand entgegenwirken, wie sie beispielsweise in Entzündungsreaktionen durch Kollagenasen oder reaktive Sauerstoffradikale ausgelöst wird. Interessanterweise hat sich das Antidiabetikum Metformin rezent als vielversprechende Therapieoption entwickelt, dessen pleiotrope Wirkmechanismen auch die Entzündungshemmung umfassen.

Retrospektive Studien sowie eine rezente Metaanalyse haben gezeigt, dass mit Metformin behandelte Typ-II-Diabetiker langsamere AAA-Progressionsraten aufweisen und weniger wahrscheinlich ein AAA entwickeln als Nichtdiabetiker [37-40]. Dieser Effekt wurde bei anderen antidiabetisch wirksamen Arzneimitteln nicht beobachtet und so konnte in einer rezenten prospektiven Kohortenstudie gezeigt werden, dass bei mit Metformin behandelte Diabetes Typ-II-Patienten die Mortalität bezogen auf die AAARupturrate als auch die Notwendigkeit einer vorzeitigen Operation signifikant vermindert ist im Vergleich zu Patienten mit anderer antidiabetischen Medikation [41]. Die Entwicklung von AAA ist auch im Mausmodell signifikant reduziert, wenn normoglykämische Mäuse mit Metformin behandelt werden [37]. Aktuell wird in zwei europäischen Studien, dem schwedischen MAAAGI (Metformin for Abdominal Aortic Aneurysm Growth Inhibition) Trial (offen randomisierte, kontrollierte Studie) und dem von unserem Studienteam in Österreich initiierten Vienna MetAAA Trial, einer prospektiven, doppelblinden, randomisierten und placebokontrollierten Sicherheits- und Wirksamkeitsstudie, die
Hypothese getestet, dass Metformin die AAA-Progression bei nichtdiabetischen Patienten reduziert [42, 43].

Insgesamt werden 170 nichtdiabetische Patienten mit einem infrarenalen abdominellen Aortenaneurysma von 30 bis $49 \mathrm{~mm}$ Durchmesser für die österreichische MetAAA-Studie rekrutiert. Nach einer zweiwöchigen Testphase (mit zunehmender Dosierung von Metformin), um Patienten mit Metformin verursachten Nebenwirkungen auszuschließen, werden die verbleibenden Patienten randomisiert und 12 Monate lang mit Placebo oder Metformin in einer Dosis von 2000 mg pro Tag behandelt. Mittels computertomographischer Angiographie werden in Intervallen von 6 Monaten das AAA-Wachstum, also der maximale Aortendurchmesser sowie das Aneurysmavolumen, bestimmt.

Interne präliminäre Daten zeigen bereits vielversprechende Ergebnisse und lassen hoffen, dass Metformin ein wissenschaftlich hochinteressantes und aussichtsreiches Medikament zur Begrenzung der AAA-Progression darstellen kann.

\section{Fazit für die Praxis}

Bisher konnte keine Arzneimittelgruppe oder einzelnes Medikament mit der Wirksamkeit zur Wachstumsreduktion beim abdominellen Aortenaneurysma wissenschaftlich nachgewiesen werden. Aus diesem Grund enthalten die aktuellen Clinical Practice Guidelines der European Society for Vascular Surgery (ESVS) als auch die Practice Guidelines der Society for Vascular Surgery diesbezüglich keine Empfehlungen zur medikamentösen Therapie des AAA. Aktuelle Studien, darunter das Vienna MetAAA Trial, werden wichtige Evidenz liefern, ob Metformin die hohen internationalen Erwartungen erfüllen und zukünftig als Medikament bei Patienten mit AAA einen zentralen Stellenwert einnehmen kann.

Die gegenwärtige klinische Praxis sieht vor allem die Reduktion des allgemeinen kardiovaskularen Risikos bei Patienten mit AAA vor. Dies inkludiert meist sogenannte Lifestyle-Veränderungen wie Raucherentwöhnung, angeleite- tes körperliches Training und gesunde Ernährung. Als konservative medikamentöse Therapie (zur allgemeinen Reduktion des kardiovaskulären Risikos bei AAA-Patienten) stehen, neben einer optimalen Blutdruckeinstellung, Thrombozytenaggregationshemmer sowie HMG-CoA-Reduktase-Inhibitoren (Statine) zur Verfügung.

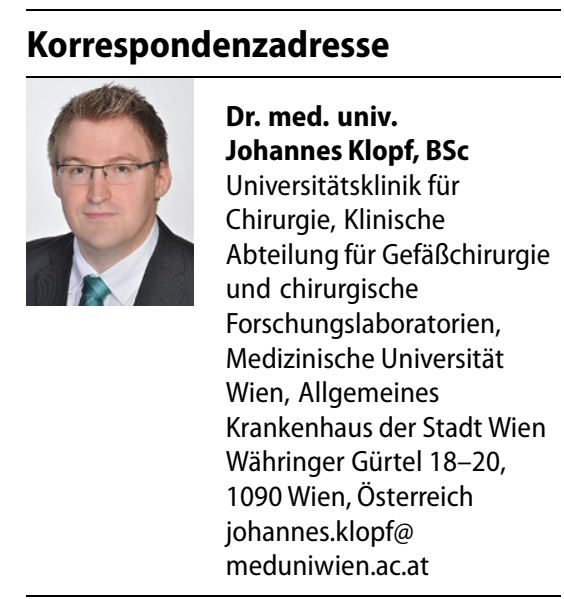

Funding. Open access funding provided by Medical University of Vienna.

\section{Einhaltung ethischer Richtlinien}

Interessenkonflikt. J. Klopf, A. Scheuba, C. Brostjan, C. Neumayer und W. Eilenberg geben an, dass keine finanziellen Interessenkonflikte bestehen.

Für diesen Beitrag wurden von den Autoren keine Studien an Menschen oder Tieren durchgeführt. Für die aufgeführten Studien gelten die jeweils dort angegebenen ethischen Richtlinien.

Open Access. Dieser Artikel wird unter der Creative Commons Namensnennung 4.0 International Lizenz veröffentlicht, welche die Nutzung, Vervielfältigung, Bearbeitung, Verbreitung und Wiedergabe in jeglichem Medium und Format erlaubt, sofern Sie den/die ursprünglichen Autor(en) und die Quelle ordnungsgemäß nennen, einen Link zur Creative Commons Lizenz beifügen und angeben, ob Änderungen vorgenommen wurden.

Die in diesem Artikel enthaltenen Bilder und sonstiges Drittmaterial unterliegen ebenfalls der genannten Creative Commons Lizenz, sofern sich aus der Abbildungslegende nichts anderes ergibt. Sofern das betreffende Material nicht unter der genannten Creative Commons Lizenz steht und die betreffende Handlung nicht nach gesetzlichen Vorschriften erlaubt ist, ist für die oben aufgeführten Weiterverwendungen des Materials die Einwilligung des jeweiligen Rechteinhabers einzuholen.

Weitere Details zur Lizenz entnehmen Sie bitte der Lizenzinformation auf http://creativecommons.org/ licenses/by/4.0/deed.de. 


\section{Literatur}

1. Bobadilla JL(2013) From EberstoEVARs:a historical perspective on aortic surgery. Aorta (Stamford) 1:89-95

2. Sweeting MJ, Thompson SG, Brown LC, Powell JT, RESCAN collaborators (2012) Meta-analysis of individual patient data to examine factors affecting growth and rupture of small abdominal aortic aneurysms. Br J Surg 99:655-665

3. Norman PE, Curci JA (2013) Understanding the effects of tobacco smoke on the pathogenesis of aortic aneurysm. Arterioscler Thromb Vasc Biol 33:1473-1477

4. Wanhainen A, Verzini F, Van Herzeele l et al (2019) Editor's choice; European society for vascular surgery (ESVS) 2019 clinical practice guidelines on the management of abdominal aorto-iliac artery aneurysms. Eur JVasc Endovasc Surg 57:8-93

5. Myers J, McElrath M, Jaffe A et al (2014) A randomized trial of exercise training in abdominal aortic aneurysm disease. Med Sci Sports Exerc 46:2-9

6. Kato M, Kubo A, Green FN, Takagi H (2019) Meta-analysis of randomized controlled trials on safety and efficacy of exercise training in patients with abdominal aortic aneurysm. J Vasc Surg 69:933-943

7. Kokje VB, Hamming JF, Lindeman JH (2015) Editor's choice - pharmaceutical management of small abdominal aortic aneurysms: a systematic review of the clinical evidence. Eur J Vasc Endovasc Surg 50:702-713

8. Bhak RH, Wininger M, Johnson GR et al (2015) Factors associated with small abdominal aortic aneurysm expansion rate. JAMA Surg 150:44-50

9. Hackam DG, Thiruchelvam D, Redelmeier DA (2006) Angiotensin-converting enzyme inhibitors and aortic rupture: a population-based casecontrol study. Lancet 368:659-665

10. Wilmink $A B$, Vardulaki $K A$, Hubbard $C S$ et al (2002) Are antihypertensive drugs associated with abdominal aortic aneurysms? J Vasc Surg 36:751-757

11. Gadowski GR, Pilcher DB, Ricci MA (1994) Abdominal aortic aneurysm expansion rate: effect of size and beta-adrenergic blockade. J Vasc Surg 19:727-731

12. Lindholt JS, Henneberg EW, Juul $S$, Fasting $H$ (1999) Impaired results of a randomised double blinded clinical trial of propranolol versus placebo on the expansion rate of small abdominal aortic aneurysms. Int Angiol 18:52-57

13. Propanolol Aneurysm Trial Investigators (2002) Propranolol for small abdominal aortic aneurysms: results of a randomized trial.J Vasc Surg 35:72-79

14. Kortekaas KE, Meijer CA, Hinnen JWetal (2014) ACE inhibitors potently reduce vascular inflammation, results of an open proof-of-concept study in the abdominal aortic aneurysm. PLoSOne 9:e111952

15. Thompson AR, Cooper JA, Ashton HA, Hafez $\mathrm{H}$ (2010) Growth rates of small abdominal aortic aneurysms correlate with clinical events. Br J Surg 97:37-44

16. Sweeting MJ, Thompson SG, Brown LC, Greenhalgh RM, Powell JT (2010) Use of angiotensin converting enzyme inhibitors is associated with increased growth rate of abdominal aortic aneurysms. JVasc Surg 52:1-4

17. Hogh A, Vammen $S$, Ostergaard L, Joensen JB, Henneberg EW, Lindholt JS (2009) Intermittent roxithromycin for preventing progression of smal abdominal aortic aneurysms: long-term results of a small clinical trial. Vasc Endovascular Surg 43:452-456

18. Karlsson L, Gnarpe J, Bergqvist D, Lindback J, Parsson H (2009) The effect of azithromycin and chlamydophilia pneumonia infection on expansion of small abdominal aortic aneurysms-a prospective randomized double-blind trial. J Vasc Surg 50:23-29

19. Mosorin M, Juvonen J, Biancari F et al (2001) Use of doxycycline to decrease the growth rate of abdominal aortic aneurysms: a randomized, double-blind, placebo-controlled pilot study. JVasc Surg 34:606-610

20. Baxter BT, Pearce WH, Waltke EA et al (2002) Prolonged administration of doxycycline in patients with small asymptomatic abdominal aortic aneurysms: report of a prospective (phase II) multicenter study.J Vasc Surg 36:1-12

21. Dunne JA, Bailey MA, Griffin KJ, Sohrabi S, Coughlin PA, Scott DJ (2014) Statins: the holy grail of abdominal aortic aneurysm (AAA) growth attenuation? A systematic review of the literature. Curr Vasc Pharmacol 12:168-172

22. Yoshimura K, Morikage N, Nishino-Fujimoto $S$, Furutani A, Shirasawa B, Hamano K (2018) Current status and perspectives on pharmacologic therapy for abdominal aortic aneurysm. Curr Drug Targets 19:1265-1275

23. Sukhija R, Aronow WS, Sandhu R, Kakar P, Babu S (2006) Mortality and size of abdominal aortic aneurysm at long-term follow-up of patients not treated surgically and treated with and without statins. Am J Cardiol 97:279-280

24. Schouten O, van Laanen JH, Boersma E et al (2006) Statins are associated with a reduced infrarenal abdominal aortic aneurysm growth. Eur J Vasc Endovasc Surg 32:21-26

25. Schlosser FJ, Tangelder MJ, Verhagen $\mathrm{HJ}$ et al (2008) Growth predictors and prognosis of small abdominal aortic aneurysms. J Vasc Surg 47:1127-1133

26. Karlsson L, Bergqvist D, Lindback J, Parsson H (2009) Expansion of small-diameter abdominal aortic aneurysms is not reflected by the release of inflammatory mediators IL-6, MMP-9 and CRP in plasma. Eur JVasc Endovasc Surg 37:420-424

27. Mosorin M, Niemela E, Heikkinen J et al (2008) The use of statins and fate of small abdominal aortic aneurysms. Interact CardioVasc Thorac Surg 7:578-581

28. Ferguson CD, Clancy P, Bourke B et al (2010) Association of statin prescription with small abdominal aortic aneurysm progression. Am Heart J 159:307-313

29. Thompson A, Cooper JA, Fabricius M, Humphries SE, Ashton HA, Hafez H (2010) An analysis of drug modulation of abdominal aortic aneurysm growth through 25 years of surveillance. J Vasc Surg 52:55-61.e2

30. Takagi H, Yamamoto H, Iwata K, Goto S, Umemoto T, Group A (2012) Effects of statin therapy on abdominal aortic aneurysm growth: a metaanalysis and meta-regression of observational comparative studies. Eur J Vasc Endovasc Surg 44:287-292

31. Pan Z, Cui H, Wu N, Zhang H (2020) Effect of statin therapy on abdominal aortic aneurysm growth rate and mortality: a systematic review and metaanalysis. Ann Vasc Surg. https://doi.org/10.1016/j. avsg.2020.03.036

32. Chaikof EL, Dalman RL, Eskandari MK et al (2018) The society forvascular surgery practice guidelines on the care of patients with an abdominal aortic aneurysm. JVasc Surg 67:2-77.e2
33. Aboyans V, Ricco JB, Bartelink MEL et al (2018) 2017 ESC guidelines on the diagnosis and treatment of peripheral arterial diseases, in collaboration with the European society for vascular surgery (ESVS): document covering atherosclerotic disease of extracranial carotid and vertebral, mesenteric, renal, upper and lower extremity arteriesendorsed by: the European stroke organization (ESO) the task force for the diagnosis and treatment of peripheral arterial diseases of the European society of cardiology (ESC) and of the European society for vascular surgery (ESVS). Eur Heart J39:763-816

34. Piepoli MF, Hoes AW, Agewall S et al (2016) 2016 European guidelines on cardiovascular disease prevention in clinical practice: the sixth joint task force of the European society of cardiology and other societies on cardiovascular disease prevention in clinical practice (constituted by representatives of 10 societies and by invited experts) developed with the special contribution of the European association for cardiovascular prevention \& rehabilitation (EACPR). Eur Heart J 37:2315-2381

35. Lindholt JS, Sorensen HT, Michel JB, Thomsen HF, Henneberg EW (2008) Low-dose aspirin may prevent growth and later surgical repair of medium-sized abdominal aortic aneurysms. Vasc Endovascular Surg 42:329-334

36. Bahia SS, Vidal-Diez A, Seshasai SR et al (2016) Cardiovascular risk prevention and all-cause mortality in primary care patients with an abdominal aortic aneurysm. Br JSurg 103:1626-1633

37. Fujimura N, Xiong J, Kettler EB et al (2016) Metformin treatment status and abdominal aortic aneurysm disease progression. J Vasc Surg 64:46-54.e8

38. Golledge J, Moxon J, Pinchbeck J et al (2017) Association between metformin prescription and growth rates of abdominal aortic aneurysms. $\mathrm{Br} J$ Surg 104:1486-1493

39. Itoga NK, Rothenberg KA, Suarez P et al (2019) Metformin prescription status and abdominal aortic aneurysm disease progression in the U.S veteran population. J Vasc Surg 69:710-716.e3

40. Yu X, Jiang D, Wang J et al (2019) Metformin prescription and aortic aneurysm: systematic review and meta-analysis. Heart 105:1351-1357

41. Golledge J, Morris DR, Pinchbeck J et al (2019) Editor's choice-metformin prescription is associated with a reduction in the combined incidence of surgical repair and rupture related mortality in patients with abdominal aortic aneurysm. Eur J Vasc Endovasc Surg 57:94-101

42. Metformin for Abdominal Aortic Aneurysm Growth Inhibition (MAAAGI). https://clinicaltrials.gov/ct2/ show/NCT04224051.Zugegriffen: 11.07.2020

43. Metformin Therapy in Non-diabetic AAA Patients (MetAAA). https://clinicaltrials.gov/ct2/show/ NCT03507413.Zugegriffen:11.07.2020 